\title{
DISTRIBUIÇÃO DO SISTEMA RADICULAR DO MILHO EM TERRA ROXA ESTRUTURADA LATOSSÓLICA: I. COMPARAÇÃO DE METODOLOGIAS ${ }^{1}$
}

\author{
L. FANTE JR2; K. REICHARDT ${ }^{3}$ \\ Centro de Energia Nuclear na Agricultura-USP, C.P. 96 - CEP: 13400-970 - Piracicaba,SP e Departamento de Flsica- \\ ESALOUSP, C.P. 9, CEP: 13418-900 - Piracicaba,SP \\ L.A.C. JORGE; S. CRESTANA \\ CNPDIA/EMBRAPA, C.P. 741, CEP: 13560-970 - Säo Carlos,SP
}

RKSUMO: A distribuição do sistema radicular do milho (Zea mays,L.) em terra roxa estruturada foi avaliada pelos métodos do trado, do cilindro volumétrico e de processamento de imagens, esse último um novo método auxiliar no estudo destes sistemas. A amostragem do sistema radicular foi definida num plano perpendicular à linha da cultura, em regiōes de $20 \times 20 \mathrm{~cm}$, abrangendo uma área total de $100 \times 100 \mathrm{~cm}$. Os resultados da quantificação radicular mostraram uma maior concentraçāo de raizes ma camada de $0-20 \mathrm{~cm}$, cerca de $70 \%$ de massa seca para uma presença radicular de aproximadamente $40 \%$. O método de processamento de imagens mostron-se como uma ferramenta poderosa no estudo detalhado de sistemas radiculares.

Descritores: processamento de imagens, sistema radicular

\section{DISTRIBUTION OF MAIZE ROOT SYSTLM IN A KANDUIDALFIC EUTRUDOX SOIL: I. COMPARISON OF METHODOLOGIES}

\begin{abstract}
Root distribution and soil-root interface were evaluated for a maize crop grown on an alfisol (kanduidalfic eutrudox soil). Soil anger, volumetric cylinder and image processing methods were used, the last one being a new auxiliary method for the study of these systems. Samples for analysis were collected in a $100 \mathrm{X} 100 \mathrm{~cm}$ grid, on a plane perpendicular to crop lines, with $20 \times 20 \mathrm{~cm}$ sub samples, totalizing 25 areas of $400 \mathrm{~cm}^{2}$. Results indicate a greater root concentration in the upper $0-20 \mathrm{~cm}$, representing about $70 \%$ of dry root matter for a root presence of about $40 \%$. The image processing method indicated to be a powerful method for the detailed study of roots systems.
\end{abstract}

Kegs words: image processing, root system

\section{INTRODUÇÃO}

A avaliação da distribuição radicular de uma cultura é fundamental para o desenvolvimento de práticas racionais de manejo agrícola que visam a otimização da produtividade. Um conhecimento detalhado do sistema radicular e das influências dos parâmetros físicos, químicos e biológicos do solo no desenvolvimento das raízes, $\epsilon$ de grande importância.

Existem vários métodos que permitem investigar o desenvolvimento do sistema radicular (BOHM, 1979; KOPKE, 1981 e SCHUURMAN \&
GOEDWAAGEN, 1971). A avaliação em termos de volume explorado, comprimento de raiz, atividade radicular, etc, é tarefa difícil e grandes dificuldades são encontradas em qualquer técnica de amostragem, como o tempo gasto, a pouca informação obtida e a grande variabilidade dos resultados.

As técnicas atualmente disponíveis não atendem de maneira satisfatória às reais necessidades, tal como uma avaliação mais completa possível do sistema radicular e uma visão clara da interface solo-raiz. E necessário que a avaliação do sistema radicular seja feita através de um número

\footnotetext{
'Trabalho realizado com o auxílio da FAPESP.

2 Bolsista da FAPESP.

${ }^{3}$ Bolsista do CNPq.
}

Sci. agric., Piracicaha, 51(3):513-518, set./dez. 1994 
relevante de grandezas, exigindo a aplicação simultânea de diferentes métodos, e, também, que essa avaliação seja feita em acoplamento à avaliação dos parâmetros físicos, químicos e biológicos do solo. Portanto, é de fundamental importância o desenvolvimento de novas metodologias para um estudo detalhado de raízes.

CRESTANA et al. (1994) apresentaram

um novo método auxiliar para o estudo do sistema radicular através da técnica de processamento de imagens. Esse método tem proporcionado resultados animadores, com boa precisão na quantificação das raízes, bem como indica permitir um estudo amplo da interface solo-raiz.

Com a preocupação de expandir a aplicação desse novo método, do seu aperfeiçoamento e ampliação e de sua comparação com demais, esse trabalho teve como objetivo central a avaliação do sistema radicular através de diferentes métodos, incluíndo o processamento de imagens.

\section{MATERIAL E MÉTODOS}

A cultura escolhida para a avaliação radicular foi a do milho (Zea mays, L.) cultivado em terra roxa estruturada, classificada como Rhodic Kandiudalf. Os métodos empregados foram o de processamento de imagens, 0 do trado e 0 denominado cilindro volumétrico.

A amostragem do sistema radicular foi definida num perfil de solo perpendicular à cultura, com posição central do eixo da planta, em regiões de $20 \times 20 \mathrm{~cm}$, abrangendo uma área total de 100 cm de largura por $100 \mathrm{~cm}$ de profundidade, com abertura de trincheira para os métodos de processamento de imagens $e$ do cilindro volumétrico. Para o método do trado, as amostras do tipo deformada com massas entre 300 a 700 gramas, foram retiradas por um trado manual e, portanto, sem abertura de trincheira, sendo duas amostras por região de $20 \times 20 \mathrm{~cm}$. A figura 1 mostra um esquema da área radicular amostrada.

Com relação ao método do cilindro volumétrico, coletaram-se amostras indeformadas cravando-se, num perfil de solo, cilindros de aproximadamente $5 \mathrm{~cm}$ de diâmetro por $10 \mathrm{~cm}$ de altura. Foram retiradas em torno de três amostras por região de $20 \times 20 \mathrm{~cm}$.

As porções de solo e raíz das amostras obtidas por trado e por cilindro volumétrico, foram separadas e levadas à estufa para a determinação de suas massas secas.
O preparo do perfil do solo para a obtenção de imagens adequadas, nas quais as raízes apresentam-se de forma mais nítida possível com relação ao solo, constituiu-se em pintar o perfil com "spray" de cor branca e posteriormente lavá-lo com jato de água. As imagens foram obtidas nas áreas de $20 \times 20 \mathrm{~cm}$ via filmadora de vídeo comum e em seguida digitalizadas e processadas.

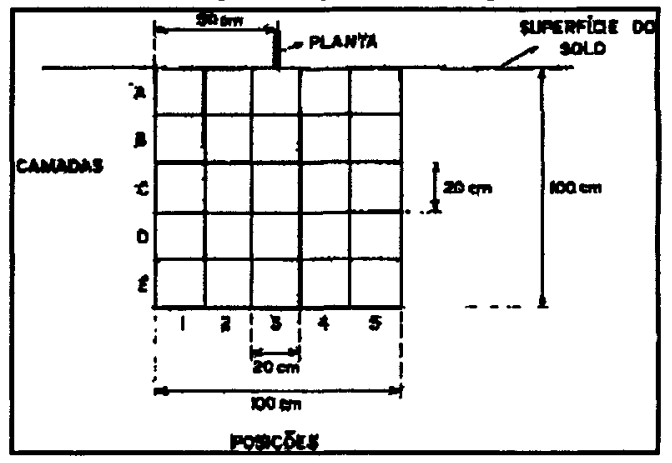

Figura 1. Desenho esquemático das regiōes amostradas para a avaliação radicular. Área total de $100 \times 100 \mathrm{~cm}$ dividida em regiões de $20 \times 20 \mathrm{~cm}$ (regiões definidas por camadas de $\mathrm{A}$ a $\mathrm{E}$ em profundidade e por diferentes posições de 1 a 5 relativas à planta).

A quantificação radicular foi feita utilizando-se da massa seca de raiz e das seguintes relações: "densidade radicular por massa - DRM" (massa seca de raiz por massa seca de solo), para os métodos do trado e do cilindro volumétrico e "densidade radicular por presença de raiz - DRPR" (razão entre o número de regiões com presença de raiz e o número total de regiōes analisadas na imagem digitalizada), para o método de processamento de imagens. Cada imagem digitalizada, correspondente à uma área de $20 \times 20 \mathrm{~cm}$, foi dividida em regiões de $1 \times 1 \mathrm{~cm}$, sendo que nessas regiões é que foi verificada a presença de raiz. Tal verificação foi feita com base em tons de cores préestabelecidos, os quais representavam as raízes. Se numa dada região de $1 \times 1 \mathrm{~cm}$ for detectada uma ou mais tonalidades características, nela é acusada a presença de raízes. O resultado final pode ser apresentado através de um histograma e/ou de forma gráfica (figura 2), e a partir desses determina-se a DRPR. A aplicação do método do cilindro volumétrico tamberm permitiu determinar simultaneamente a densidade global do solo, para as respectivas regiões e camadas amostradas. Isto 
foi possível uma vez que as amostras eram do tipo indeformada. Entretanto, o volume radicular contido na amostra, foi estimado e descontado do volume total para a obtenção do volume de solo. Estimou-se o volume radicular, considerando-se o mesmo igual a massa da raíz saturada com água, pois nesta condição $\varepsilon$ satisfatório admitir que praticamente toda massa medida, ou seja, todo volume, $\epsilon$ devido à água contida na raiz. É claro que essa estimativa proporciona considerável incerteza no volume radicular, contudo, sabendo-se que esse volume $\epsilon$ necessariamente desprezível quando comparado com o volume de solo amostrado, o valor final da densidade global do solo certamente se apresentará com pequena incerteza.

(a)

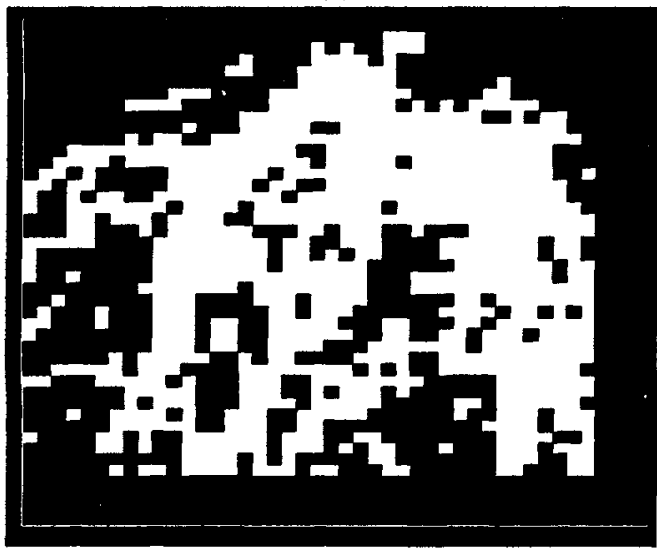

(b)

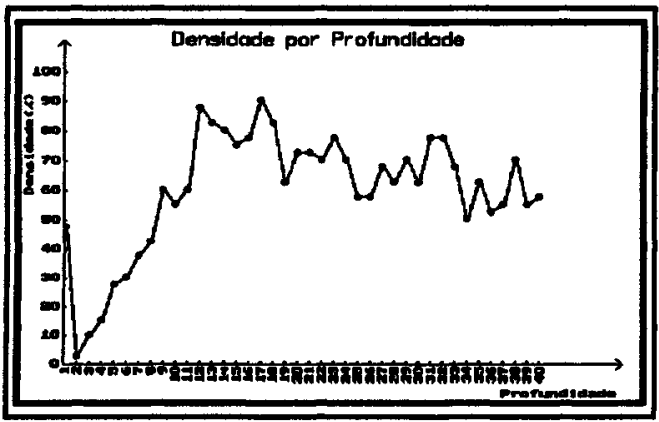

Figura 2. (a) Histograma de saida referente a uma dada região de $20 \times 20 \mathrm{~cm}$, dividida em pequenas regiōes de $1 \times 1 \mathrm{~cm}$. Cada uma das regiōes pintada indica presença de raiz. (b) Grafico de densidade radicular (em \%) em função da profundidade referente a um dado histograma de saída.

\section{RESULTADOS E DISCUSSÃO}

Na TABELA 1 são apresentados os valores de densidade global e os dados encontrados da análise mecânica, para as diferentes camadas do perfil de solo.

TABELA 1. Valores de densidade global e da análise granulométrica do solo, para as diferentes camadas. Os valores de densidade global do solo são médias de três repetições (três amostras retiradas via anéis de Kopeck).

\begin{tabular}{cccccc}
\hline $\begin{array}{c}\text { camada } \\
(\mathrm{cm})\end{array}$ & $\begin{array}{c}\text { dens. do } \\
\text { solo } \\
\left(\mathrm{g} / \mathrm{cm}^{3}\right)\end{array}$ & $\begin{array}{c}\text { argila limo } \\
(\%)\end{array}$ & $(\%)$ & $\begin{array}{c}\text { areia } \\
\text { grossa } \\
(\%)\end{array}$ & $\begin{array}{c}\text { areia } \\
\text { fina } \\
(\%)\end{array}$ \\
\hline $0-20$ & 1,419 & 36,8 & 31,9 & 24,4 & 6,9 \\
$20-35$ & 1,537 & 43,8 & 34,5 & 19,6 & 2,1 \\
$35-50$ & 1,467 & 43,1 & 38,8 & 13,9 & 4,2 \\
$50-65$ & 1,477 & 57,6 & 20,7 & 16,6 & 5,1 \\
$65-75$ & 1,420 & 54,5 & 19,8 & 19,5 & 6,2 \\
$75-85$ & 1,270 & 55,4 & 15,2 & 21,8 & 7,6 \\
$85-100$ & 1,247 & 53,2 & 19,2 & 20,6 & 7,0 \\
$100-115$ & 1,300 & 53,2 & 18,2 & 21,2 & 7,4 \\
\hline
\end{tabular}

Os resultados de massa seca de raiz, referentes à aplicação dos métodos do trado e do cilindro volumétrico, são mostrados na TABELA 2. Os valores apresentados são quantidade total de massa retirada por camada e a contribuiçâo percentual dessa relativo à massa resultante obtida para toda a região amostrada $(100 \times 100 \mathrm{~cm})$.

Pode-se observar que a amostragem pelos dois métodos e para as respectivas camadas, resultou em quantidades de massa praticamente iguais, exceto para a camada de $20-40 \mathrm{~cm}$, onde a amostragem por trado indicou maior massa retirada, cerca de trés vezes mais que aquela obtida pelo cilindro volumétrico. Entretanto, deve ser ressaltado que o tamanho da amostra $e$, consequentemente a massa total retirada via trado, foram de aproximadamente duas a três vezes superiores, indicando que a amostragem por cilindro parece ser mais abrangente, proporcionando uma menor incerteza nos resultados. Por outro lado, o método do trado permitiu uma amostragem mais rápida, possibilitando um maior número de repetições em relação a profundidade $e$ a posição relativa à planta, fato também verificado por KOPKE (1981) 
na comparação entre os métodos do trado e do monolito, sendo esse último muito similar ao método do cilindro volumétrico.

TABELA 2. Valores de massa seca de raíz (em gramas e em porcentagem, para as diferentes camadas, obtidos pela aplicação dos métodos do trado e do cilindro volumétrico.

\begin{tabular}{lcccc}
\hline & \multicolumn{2}{c}{ trado } & & \multicolumn{2}{c}{ cilindro volumétrico } \\
\cline { 2 - 5 } $\begin{array}{l}\text { camada } \\
(\mathrm{cm})\end{array}$ & $(\mathrm{g})$ & $(\%)$ & $(\mathrm{g})$ & $(\%)$ \\
\hline $0-20$ & 1,2312 & 56 & 1,1751 & 72 \\
$20-40$ & $\mathbf{0 , 6 5 6 0}$ & 30 & 0,2193 & 13 \\
$40-60$ & 0,1923 & 8 & 0,1097 & 7 \\
$60-80$ & 0,0856 & 4 & 0,0840 & 5 \\
$80-100$ & 0,0388 & 2 & 0,0410 & 3 \\
\hline
\end{tabular}

Uma característica positiva na utilização do método do cilindro volumétrico, quando comparado com o do trado, é que sendo a amostra do tipo indeformada, essa pode possibilitar a determinação de um maior número de grandezas para a quantificação radicular. Contudo, considerando-se o tamanho e o tipo da raiz analisada, bem como a textura argilosa do solo em questão, o número de grandezas analisadas foi limitado.

Neste caso, exceto pela possibilidade de obtenção da densidade global do solo via método do cilindro volumétrico, o método do trado mostrou-se mais efiente, ou seja, para uma quantificação radicular rápida e simples, o método do trado pode ser considerado o mais apropriado.

Demais resultados da quantificação radicular, referentes às regiões de $20 \times 20 \mathrm{~cm}$, são apresentados na figura 3 . Essa figura mostra a distribuição espacial radicular em termos das grandezas DRM e DRPR. Pode-se observar pequenas diferenças entre as distribuições radiculares por massa relativas aos dois métodos em questão, mostrando uma boa concordância entre ambos. A maior concentração de massa foi verificada na camada de $0-20 \mathrm{~cm}$ (camada A) e na posição central da planta (posição 3), com destaque para o método do cilindro volumétrico, sendo que o método do trado mostrou considerável quantidade de massa também na camada de $20-40 \mathrm{~cm}$ (camada B).

Já a distribuição espacial radicular por presença de raiz, obtida pela aplicação do método de processamento de imagens, apresentou-se diferente das demais. Entretanto, ainda observa-se maiores concentrações de raízes nas camadas $\mathrm{A}$ e B e na posição 3.
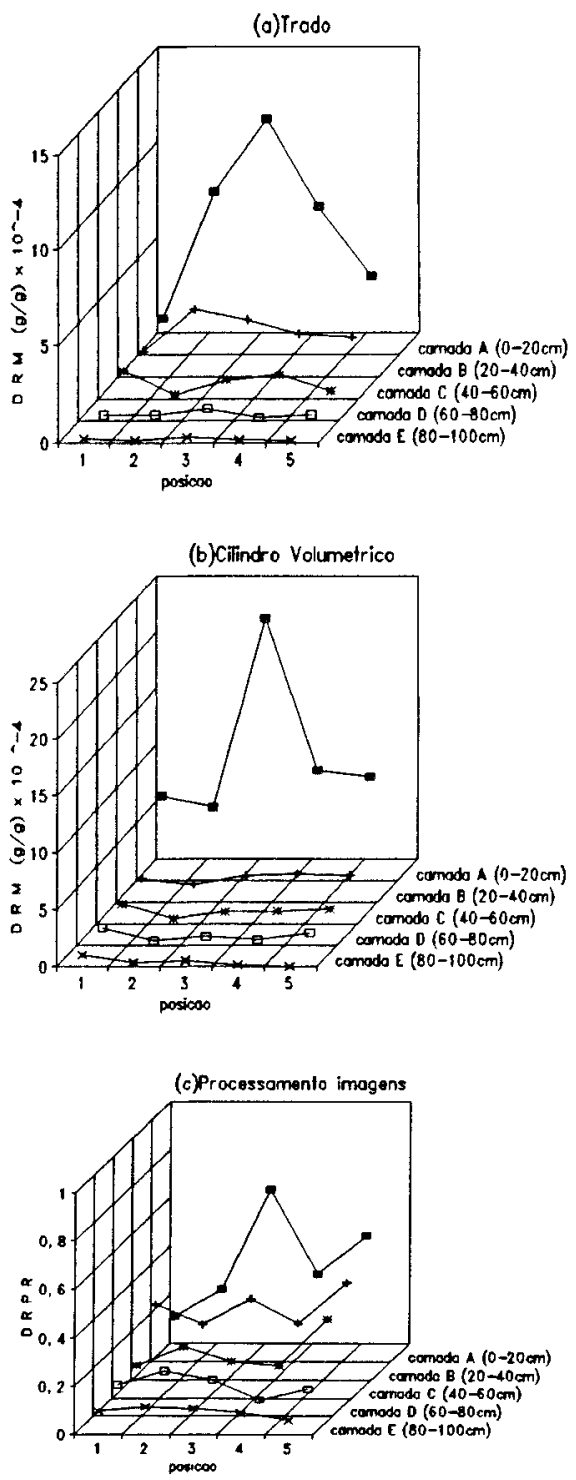

Figura 3. Distribuição espacial radicular da cultura de milho em função da camada de solo $\mathrm{c}$ da posição central da planta, para os diferentes métodos empregados: (a)do trado, (b)do cilindro volumétrico e (c)de processamento de imagens. 
A figura 4 mostra os resultados de densidade radicular de forma percentual por camada e em função da densidade radicular total calculada na área de $100 \times 100 \mathrm{~cm}$. Analisando-se essa figura, verifica-se uma boa concordância entre os métodos cuja quantificação é feita com base na massa seca de raiz, com maior concentração radicular na camada de $0-20 \mathrm{~cm}$, quase que $70 \%$ de massa seca. Novamente, nota-se uma diferença nos resultados da quantificação feita pelo método de processamento de imagens. Apesar de grande parte das raízes estarem presentes na camada A, aproximadamente $40 \%$, encontram-se quantidades relevantes de raízes nas demais camadas.

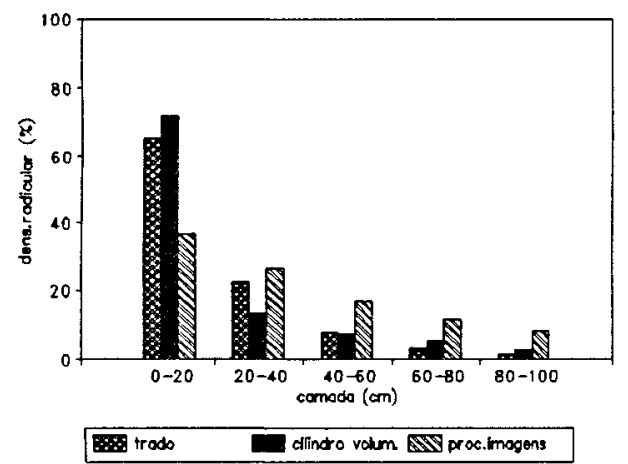

Figura 4. Distribuição radicular do milho em função da camada de solo, para os diferentes métodos empregados.

Parece ser claro que essas diferenças mencionadas já eram esperadas, uma vez que a quantificação radicular foi feita a partir de grandezas distintas. Assim, considerando-se os resultados satisfatórios, qual poderia ser utilizado para a quantificação das raízes?

Não é suficiente caracterizar o sistema radicular através de uma ou duas grandezas relevantes. Geralmente, os métodos de avaliação radicular existentes não permitem uma análise completa, e quando isto acontece, todo o processo para a quantificação $\epsilon$ complicado e demorado. Para ATKINSON (1980), uma combinação de métodos é a melhor opção em questão.

Já o método de processamento de imagens oferece uma rapidez e precisão consideráveis no estudo do sistema radicular, e permite analisá-lo de forma mais completa possível, pois esse método pode ser trabalhado e ampliado para se determinar uma quantificação radicular a partir de várias grandezas relevantes: presença de raiz, tamanho de raiz, volume e superfície radicular, quantidade de raiz, etc.. Outro aspecto importante, é que essa nova metodologia indica ajudar nos estudos sobre a interface solo-raiz, uma vez que pode ser utilizada para uma análise morfológica do solo (CRESTANA et al., 1991) e também associada a demais técnicas de obtenção e processamento de imagens, como por exemplo a tomografía computadorizada (VAZ, 1989). A amostragem para a obtenção de imagens, através de abertura de trincheiras e preparo do perfil de solo a ser filmado, não é tão simples e é feita de maneira destrutiva. Por outro lado, comparando-se com demais métodos, essa amostragem não é das mais complicadas, sendo que ainda pode ser realizada de maneira não destrutiva via o uso do minirhizotron (CRESTANA, 1990 e CHENG et al., 1991).

\section{CONCLUSÃO}

$\mathrm{Na}$ comparação entre os diversos métodos utilizados na avaliação radicular, o do trado constituiu-se no mais adequado para uma avaliação simples, rápida e barata, contudo, insuficiente para uma caracterização completa do sistema radicular. o método do cilindro volumétrico deve ser utilizado nos casos de amostragem localizadas e em pequenas regiões, permitindo um estudo quase que puntual da distribuição radicular através de um número mais significativo de grandezas e, também, permitindo correlacionar densidade radicular e densidade do solo. O método de processamento de imagens certamente se apresentou como uma ferramenta muito poderosa para o estudo detalhado do sistema radicular podendo ser também aplicado às diversas áreas dentro da ciência agronômica.

\section{REFERÊNCIAS BIBLIOGRÁFICAS}

ATKINSON, D. The distribution and effectiveness of the roots of tree crops. Horticultural Reviews, New York, v.2, p.424-490, 1980.

BOHM, W. Methods of studying root systems. New York: Springer Verlag Berlin Heidelberg, 1979, 190p.

CHENG, W.; COLEMAN, D.C.; BOX Jr, J.E. Measuring root turnover using the minirhizotron technique. Agricultural Ecosystem and Environment, v.34, n.1/4, p.261-167, 1991.

CRESTANA, S. Caracterização de raízes e compactaçāo do solo através do minirhizotron e da tomografia computadorizada. São Carlos: CNPDIA, 1990. 
CRESTANA, S.; GUIMARÃES, M.F.; JORGE, L.A.C.; RALISH, R.; TOZZI, C.L.; TORRE, A.; VAZ, C.M.P. Avaliação da distribuição de raízes no solo auxiliada por processamento de imagens digitais. Revista Brasileira de Ciencia do Solo, Campinas, v.18, n.3, p.339, 1994.

EMPRESA BRASILEIRA DE PESQUISA AGROPECUÁRIA. Serviço Nacional de Levantamento e Conservação de Solos, Rio de Janeiro. Manual de método de análise de solo, Rio de Janeiro, 1979, 227p.

KOPKE, V. Methods for studying root growth.IN: SYMPOSIUM ON THE SOIL/ROOT SYSTEM IN RELATION TO BRAZIL AGRICULTURE, Londrina, 1980. Proceedings... Londrina, Fundação Instituto Agronômico do Paraná, Londrina, 1981, p.130.
SCHUURMAN, J.J.; GOEDEWAAGEN, M.A.J. Methods for the examination of root systems and root. 2.ed. Wageningen: Pudoc, 1971, 86p.

VAZ, C.M.P. Tomografia computadorizada aplicada a estudos de compactação de solos, 1989, 110p. Dissertação (Mestrado), Escola Superior de Agricultura "Luiz de Queiroz", Universidade de São Paulo.

Recebido para publicação em 02.03 .94 Aceito para publicação em 10.03 .95 